ARTIGO ORIGINAL ORIGINAL ARTICLE

\section{Custo-utilidade da empagliflozina em pacientes diabéticos do tipo 2 com alto risco cardiovascular na perspectiva do Sistema Único de Saúde}

\author{
Cost-utility of empagliflozin in type 2 diabetic \\ patients with high cardiovascular risk in the Brazilian \\ Public Healthcare System perspective \\ Natália Bolzachini Santoni', Rodrigo Antonini Ribeiro², Ana Cláudia da \\ Cunha Travassos', Daniela Luciano Pastor Veiga' ${ }^{1}$ Thais Gomes Melo
}

DOI: 10.21115/JBES.v10.n1.p56-63

\section{Palavras-chave:}

custo-efetividade, empagliflozina, diabetes tipo 2

\section{Keywords:}

cost-effectiveness, empagliflozina, type 2 diabetes

\section{RESUMO}

Objetivo: Realizar uma análise de custo-utilidade de empagliflozina em pacientes com diabetes mellitus tipo 2 (DM2) e alto risco cardiovascular. Métodos: O modelo empregado foi uma simulação de eventos discretos, com dados baseados na incidência de eventos cardiovasculares do ensaio clínico EMPA-REG OUTCOME. A população-alvo foi de sujeitos com DM2 e alto risco cardiovascular (histórico de cardiopatia isquêmica, acidente vascular cerebral ou doença vascular periférica). 0 horizonte temporal foi o de tempo de vida; a taxa de desconto, de 5\% ao ano; e a perspectiva, a do Sistema Único de Saúde (SUS). Os dados de utilidade foram obtidos de estudos brasileiros e internacionais. Os custos refletiram valores desembolsados pelo SUS. Foram conduzidas análises de sensibilidade determinísticas nos principais parâmetros e probabilística para avaliar a robustez global dos resultados. Resultados: No caso-base, a empagliflozina gerou 0,66 ano de vida ajustados para qualidade (QALY) a mais, com custo de R\$19.176 maior, gerando relação de custo-efetividade incremental (RCEI) de R\$28.960/QALY. As análises de sensibilidade univariadas mostraram oscilações da RCEl entre R\$23.644 e R\$38.850/QALY, sendo este valor mais alto de RCEI resultante da variação do valor de utilidade de pacientes com diabetes. Na análise probabilística, os percentis 2,5\% e 97,5\% foram de R\$22.336 a R\$39.571/QALY. Conclusão: O estudo mostrou uma RCEI de R\$28.960/QALY, estando abaixo do valor de uma vez o produto interno bruto (PIB) per capita (R\$30.407 em 2016), sendo, portanto, uma tecnologia custo-efetiva, considerando esse limiar. Na análise probabilística, o intervalo de credibilidade mostrou que a RCEI pode oscilar entre 0,73 e 1,30 PIB per capita, sendo, portanto, uma estimativa robusta.

\footnotetext{
Recebido em: 27/02/2018. A provado para publicação em: 23/03/2018.

1. Boehringer Ingelheim, São Paulo, SP, Brasil.

2. HTAnalyze Economia e Gestão em Saúde, Porto Alegre, RS, Brasil.

Financiamento: Este estudo foi financiado pela Boehringer Ingelheim do Brasil, instituição onde o trabalho foi executado. Este estudo não foi apresentado previamente em congressos

Autor correspondente: Natália Bolzachini Santoni. Av. das Nações Unidas, 14171, Torre B, $18^{\circ}$ andar, Santo Amaro, São Paulo, SP. CEP: 04794-000. Telefone: (11) 4949-4955. E-mail: natalia.santoni@boehringer-ingelheim.com
} 
0.66 QALY and R\$ 19,176, resulting in an incremental cost-effectiveness ratio (ICER) of R\$ 28,960/ QALY. Univariate sensitivity analysis showed variations in the ICER between $\mathrm{R} \$ 23,644$ and $\mathrm{R} \$ 38,850 /$ QALY, with the diabetes status utility the variable with most influence in the ICER. In the probabilistic sensitivity analysis, 2.5\% and 97.5\% percentiles were R\$22,336 and R\$39,571/QALY. Conclusion: The study showed an ICER of R\$28,960/QALY, which is below the value of one GDP per capita in Brazil (R\$30,407 in 2016), which would therefore be deemed cost-effective under this threshold. In probabilistic sensitivity analysis, credible interval ranged between 0.73 and 1.30 per capita GDP, therefore showing robust results.

\section{Introdução}

Estimativas dos últimos anos mostram que 415 milhões de adultos são portadores de diabetes mellitus tipo 2 (DM2); no Brasil, em 2013, havia 11.934.000 de adultos diabéticos, número esse que aumenta a cada ano. A Pesquisa Nacional de Saúde de 2013 mostrou prevalência da doença de 6,2\% na população em geral, aumentando para quase 20\% na população acima de 75 anos (Iser et al., 2015). O impacto do diabetes em termos de saúde pública é muito grande no Brasil. Em estudo publicado em 2014, foi estimado que entre 8\% e 12\% de todas as internações hospitalares no Sistema Único de Saúde (SUS) estão ligadas de forma direta ou indireta ao diabetes (Rosa et al., 2014). Os custos consequentes do diabetes são elevados. Em estimativa de 2011, foi calculado que o custo de seguimento ambulatorial de pacientes com diabetes era de US\$ 2.108 anuais, dos quais cerca de dois terços eram custos diretos. Pacientes com complicações micro ou macrovasculares tinham os custos mais elevados, quase $50 \%$ acima dessa média (Bahia et al., 2011).

No SUS, o tratamento usual inclui medicamentos das classes das biguanidas e sulfonilureias, além de insulina. Outras classes de medicamentos, como os das classes de inibidores da a-glucosidase, glinidas, glitazonas, inibidores de SGLT2, agonistas do GLP-1 e inibidores da DPP4, não estão incorporadas no SUS. A empagliflozina é um medicamento pertencente a uma nova classe de medicamentos orais para o tratamento de DM2, os inibidores de SGLT2, que atuam inibindo os cotransportadores de sódio-glicose do túbulo proximal renal, impedindo a reabsorção renal de glicose e, dessa forma, reduzindo a hiperglicemia. O principal ensaio clínico que avaliou essa medicação em pacientes com doença cardiovascular estabelecida foi o EMPA-REG OUTCOME, no qual 7.020 pacientes diabéticos e com alto risco cardiovascular, já em tratamento com metformina, sulfonilureias e/ou insulina, foram randomizados para empagliflozina ou placebo e seguidos por um tempo médio de três anos (Zinman et al., 2015). O estudo mostrou redução de eventos cardiovasculares e mortalidade, com um hazard ratio (HR) para o desfecho primário de morte cardiovascular, infarto agudo do miocárdio (IAM) ou acidente vascular cerebral (AVC) de 0,86 (intervalo de confiança [IC] 95\%: 0,74-0,99), o qual teve como maior peso a morte cardiovascular, que foi reduzida em 38\%. Também foi verificado no mesmo estudo redução de eventos associados à nefropatia e insuficiência cardíaca (ICC). Alguns desses desfechos mostraram maior redução de eventos nas análises de subgrupo por faixa etária, com benefício superior em pacientes acima de 65 anos.

No Brasil, assim como em vários outros países, a incorporação de tecnologias requer cada vez mais um uso criterioso de recursos econômicos, sendo etapa fundamental da avaliação de tecnologias em saúde as avaliações farmacoeconômicas. Neste estudo, o objetivo foi de avaliar a relação de custo-efetividade de empagliflozina adicionada ao cuidado usual (metformina, sulfonilureia e/ou insulina) versus apenas cuidado usual, em pacientes do SUS.

\section{Métodos}

\section{Descrição do modelo econômico}

O modelo desenvolvido é uma simulação de eventos discretos que compara os custos e consequências de tratamento padrão para o DM2 versus o mesmo com adição de empagliflozina. 0 modelo inicia com a criação de perfis de pacientes simulados, os quais representam população semelhante à estudada no estudo EMPA-REG OUTCOME (Zinman et al., 2015). Esse estudo é o principal ensaio clínico de empagliflozina em pacientes com doença cardiovascular estabelecida, sendo o que, além de ter o maior tempo de seguimento entre todos os trabalhos sobre essa medicação, também é o com a maior tamanho de população e o único efetivamente desenhado para mensurar a ocorrência de desfechos clinicamente relevantes (especialmente eventos cardiovasculares), sendo, por esse motivo, selecionado como a fonte de evidência clínica. O modelo utiliza diretamente os dados do EMPA-REG OUTCOME para simular o tempo para eventos de uma série de diferentes condições clínicas: morte cardiovascular, ICC, insuficiência renal crônica (IRC), macroalbuminúria, instituição de hemodiálise, IAM, AVC, acidente isquêmico transitório (AIT), revascularização do miocárdio e hospitalização por angina. Foram modelados com incidência diferente entre as duas estratégias avaliadas apenas os eventos nos quais houve diferença significativa, que são os cinco primeiros eventos listados acima. Os demais eventos foram modelados, mas utilizou-se um $H R=1$, isto é, não houve 
diferença em suas taxas de incidência entre empagliflozina e tratamento padrão. Cada paciente no modelo está sujeito à ocorrência de todos esses eventos ao longo do tempo. O modelo simula cada paciente até que ele tenha progredido para óbito, quando são então computados os custos acumulados e anos de vida ganhos (AVGs), que são ajustados para qualidade (QALY), e iniciada a simulação do próximo paciente.

O modelo foi construído no software MS Excel. A representação esquemática do processamento da simulação pode ser vista na Figura 1. A seleção de perfil de paciente é feita sorteando-se um paciente entre a população do estudo EMPA-REG OUTCOME, de forma que a população terá, portanto, características semelhantes a ele: idade média de 63 anos, $71 \%$ homens, $72 \%$ brancos, índice de massa corporal (IMC) médio de $30 \mathrm{~kg} / \mathrm{m}^{2}, 75 \%$ com doença coronariana prévia, 23\% com histórico de AVC e hemoglobina glicada inicial de 8\%.

A predição de eventos é realizada por equações paramétricas, baseadas nos dados do EMPA-REG OUTCOME. Para cada um dos eventos, diferentes funções paramétricas foram testadas, sendo selecionadas as que tinham melhor ajuste dos dados: Weibull para morte cardiovascular, ICC e AVC, Gompertz para macroalbuminúria e exponencial para todos os demais eventos.

\section{Pressupostos do modelo}

A perspectiva do modelo foi a do SUS. O horizonte temporal adotado no caso-base foi o de tempo de vida (lifetime horizon) e a taxa de desconto foi de 5\% para custos e efetividade, de acordo com as recomendações das Diretrizes Metodológicas para Estudos de Avaliação Econômica de Tecnologias em Saúde (Ribeiro et al., 2016).

\section{Valores de utilidade}

Foi feita busca por valores de utilidades no cenário nacional, com foco nas condições clínicas representadas no modelo. Além de realizar busca diretamente por valores de utilidade, também foram procurados estudos que tivessem aplicado SF-36 ou EQ-5D em população nacional, uma vez que já existem fórmulas disponíveis no Brasil para fazer a adaptação desses questionários para valores de utilidade (Cruz et al., 2011, Santos et al., 2016). Os estudos localizados cujos pesquisadores disponibilizaram seus bancos de dados (o que é necessário para a aplicação das fórmulas supracitadas) são descritos a seguir. Todos eles haviam utilizado o SF-36.

- Estudo de base populacional em Campinas, com inclusão apenas de pacientes acima de 60 anos (idade média de 69,5), o qual teve em sua amostra 333 pacientes diabéticos (Lima et al., 2012). O valor médio de utilidade foi 0,792 $\pm 0,087$.

- Estudo sobre qualidade de vida em pacientes com cardiopatia isquêmica na cidade de São Paulo $(n=547)$ (Favarato et al., 2007). O valor médio de utilidade foi de 0,730 \pm 0,048 na linha de base.

- Estudo com 173 pacientes de ambulatório especializado em doença arterial coronariana no Hospital de Clínicas, Porto Alegre (Cruz et al., 2009). O valor médio de utilidade foi de 0,75 $\pm 0,073$.

- Estudo com 170 pacientes com insuficiência cardíaca de dois hospitais do estado de São Paulo, com indivíduos com média de idade de 67 anos, com cerca de $40 \%$ em classe funcional I, 40\% em classe funcional II e os demais em classes III e IV (Saccomann et al., 2010). O valor médio de utilidade foi de 0,786 $\pm 0,067$.

- Estudo com 50 pacientes do Instituto de Cardiologia (Porto Alegre) com insuficiência cardíaca, maioria homens, com idade média em torno de 60 anos e predomínio de classe funcional III (58\%) e II (38\%). O valor médio de utilidade foi de 0,732 $\pm 0,062$ (Moreira et al., 2009).

- Estudo com 223 idosos (idade média de 69,5 anos) de Belo Horizonte em tratamento dialítico, dos quais 49\% eram diabéticos. Diálise havia sido instituída há no máximo 24 meses e há no mínimo três meses. O valor médio de utilidade foi de 0,787

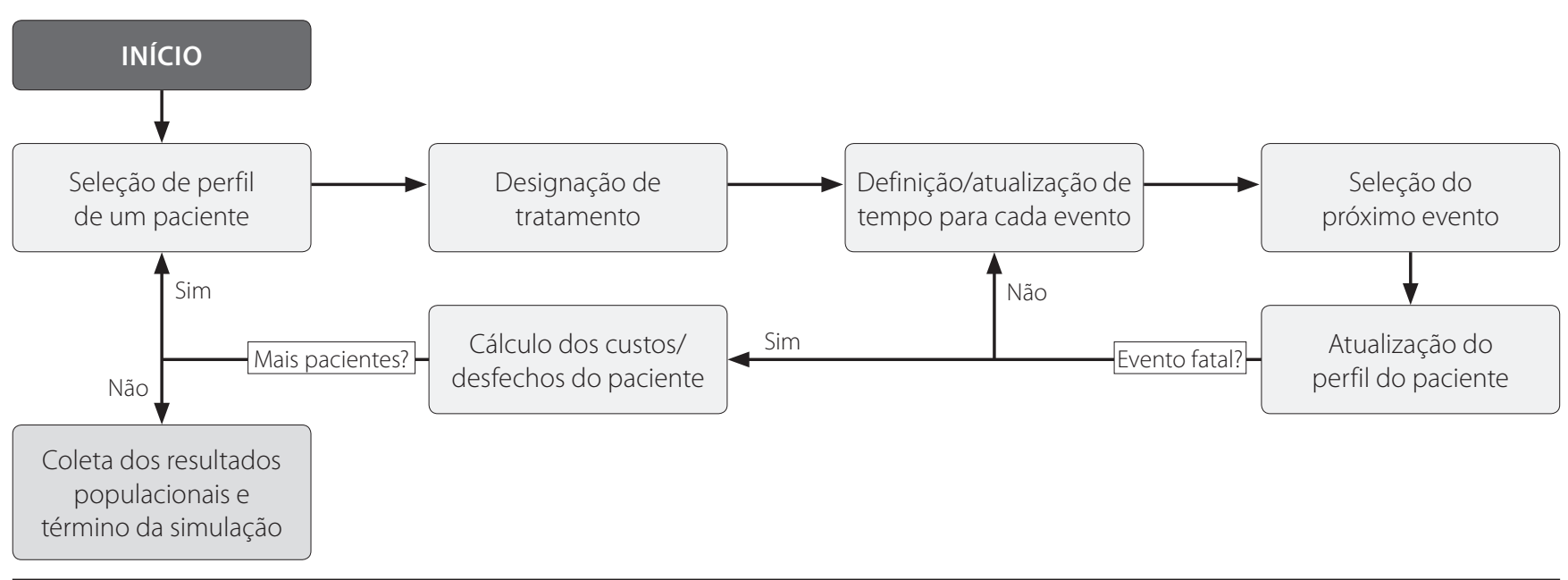

Figura 1. Representação esquemática do modelo de simulação de eventos discretos. 
$\pm 0,086$. Dividindo-se os pacientes de acordo com o status glicêmico, os valores foram relativamente parecidos $(0,767$ vs. 0,806, em diabéticos e não diabéticos, respectivamente) (Braga et al., 2011).

Para as duas condições clínicas nas quais havia mais de uma fonte de dados (cardiopatia isquêmica e insuficiência cardíaca), foi realizada metanálise de braço único para agregar os resultados em um valor médio, utilizando modelo de efeitos randômicos no software Comprehensive Meta-Analysis.

Conforme o exposto, não encontramos dados nacionais para três condições clínicas (AVC, AIT e revascularização miocárdica). Para tais estados de saúde, foi usado estudo internacional de Sullivan \& Ghushchyan, o qual avaliou 20 mil pacientes diabéticos de um grande banco de dados de quatro países (EUA, Reino Unido, França e Espanha) (Sullivan \& Ghushchyan, 2016). Nesse estudo, foram estimados valores de utilidade tanto para diabetes como para as comorbidades, no qual o efeito da comorbidade foi avaliado na própria amostra. Isto é, em vez de realizar a aferição, por exemplo, do valor médio de utilidade em uma amostra de pacientes com AVC, foi aferida a diferença de utilidade, nessa amostra, entre pacientes com diabetes sem e com essa comorbidade. Na Tabela 1, são mostrados os valores usados no modelo. Digno de nota, o estudo de Sullivan \& Ghushchyan também estimou valores de utilidade para as condições para as quais se conseguiram dados brasileiros; os valores diferiram muito pouco dos dados do Brasil (em no máximo 0,02), o que sugere ser adequado usar tal estudo para obter os dados brasileiros faltantes.

\section{Dados de custos}

O modelo trabalha com custos de eventos agudos e crônicos, uma vez que pacientes que sofrem, por exemplo, um IAM ou AVC terão custos para o manejo crônico dessas enfermidades. Conforme comentado acima, alguns pacientes já tinham tais comorbidades na linha de base. Para tais pacientes, os custos crônicos são computados desde o início. Os demais terão custos crônicos computados apenas após a ocorrência de eventos durante a simulação.

Tabela 1. Valores de utilidade usados no modelo

\begin{tabular}{ll}
\hline Condição & Valor de utilidade \\
\hline Diabetes & 0,792 \\
\hline Infarto ou angina & 0,736 \\
\hline Insuficiência cardíaca & 0,757 \\
\hline Doença renal & 0,767 \\
\hline AVC & 0,732 \\
\hline AlT & 0,722 \\
\hline Revascularização & 0,762 \\
\hline
\end{tabular}

Os custos de eventos agudos (exceto revascularização) foram buscados no Sistema de Informações Hospitalares do SUS (SIH/SUS) do Departamento de Informática do SUS (Datasus), para o período de janeiro a dezembro de 2016. As buscas foram feitas pelos Códigos Internacionais de Doenças (CIDs) correspondentes, exceto para os procedimentos de revascularização, que foram buscados pelo código de procedimento. Quando havia mais de uma fonte para a estimativa de custo, foi feita média ponderada. Os valores utilizados no modelo são mostrados na Tabela 2.

Foram considerados como custos crônicos no modelo aqueles decorrentes do cuidado ao paciente após ele desenvolver alguma das seguintes patologias: ICC, IRC com necessidade de hemodiálise, AVC, IAM ou angina ou revascularização (todas consideradas como geradoras de custos crônicos de pacientes com cardiopatia isquêmica). A fonte ideal para esse tipo de custo são publicações de coortes de pacientes brasileiros com essas condições, com quantificação e custeio de recursos utilizados no seguimento. Foram realizadas buscas na literatura com o intuito de localizar potenciais fontes para esses dados.

A patologia com maior número de artigos localizados foi a insuficiência renal terminal, já com instauração de hemodiálise (Cherchiglia et al., 2010; de Abreu et al., 2011; Ferreira, 2009; Sesso et al., 2003). Consideramos como a melhor fonte de dados o estudo com a maior casuística, baseado em levantamento de Autorização de Procedimentos de Alta Complexidade (APACs) de um grande banco de dados (10.000 pacientes). O custo médio anual aferido foi de R\$ 26.800 nos pacientes em hemodiálise e de $R \$ 33.800$ nos que estavam em diálise peritonial (Cherchiglia et al., 2010). Realizando a ponderação pela prevalência do uso de cada tipo de diálise no artigo, o valor médio seria de $\mathrm{R} \$ 27.605$. A correção pela inflação de valores em saúde é um tema controverso, uma vez que os reajustes de valores no SUS não acompanham, necessariamente, a reposição da inflação. Optamos por rea-

Tabela 2. Custos de eventos e procedimentos no Datasus - ano 2016

\begin{tabular}{|c|c|c|}
\hline Evento/procedimento & $\begin{array}{c}\text { AlHs } \\
\text { aprovadas }\end{array}$ & $\begin{array}{c}\text { Valor médio } \\
\text { da AlH }\end{array}$ \\
\hline Insuficiência cardíaca & 213.593 & $R \$ 1.560,98$ \\
\hline Insuficiência renal & 104.383 & $\mathrm{R} \$ 3.588,11$ \\
\hline Acidente isquêmico transitório & 21.001 & $\mathrm{R} \$ 1.103,89$ \\
\hline Acidente vascular cerebral* & 215.373 & $R \$ 1.877,88$ \\
\hline Infarto ou angina** & 260.341 & $\mathrm{R} \$ 4.173,12$ \\
\hline Revascularização miocárdica*** & 91.862 & $R \$ 7.820,58$ \\
\hline \multicolumn{3}{|c|}{$\begin{array}{l}\text { * CIDs utilizados na média ponderada: acidente vascular cerebral não } \\
\text { especificado como hemorrágico ou isquêmico, infarto cerebral, outras doenças } \\
\text { cerebrovasculares, hemorragia intracraniana. ** CIDs utilizados na média } \\
\text { ponderada: infarto agudo do miocárdio, outras doenças isquêmicas do coração. } \\
\text { *** Média ponderada entre todos os códigos de cirurgias de revascularização e } \\
\text { angioplastias coronarianas. AlHs: Autorização de Internação Hospitalar. }\end{array}$} \\
\hline
\end{tabular}


justar esse valor de acordo com a inflação verificada entre 2010 e 2017 no valor de hemodiálise no Sistema de Gerenciamento da Tabela de Procedimentos (SIGTAP), que foi de 34\%; com isso, o valor atualizado utilizado no modelo foi de R\$ 37.184 .

Para cardiopatia isquêmica, o artigo encontrado estimou o custo de manutenção em $\mathrm{R} \$ 2.733$, analisando uma coorte de cerca de 150 pacientres em Porto Alegre (Ribeiro et al., 2005). Na ausência de um indicador de inflação mais acurado para os valores de remuneração praticados no SUS, optou-se por reajuste pelo Índice de Preços ao Consumidor Amplo (IPCA), acumulado em um total de 98,5\%. O valor para o caso-base foi, portanto, de $\mathrm{R} \$$ 5.424. Para insuficiência cardíaca, foi localizado um artigo de 2005, cujo levantamento de dados retrospectivo de 70 pacientes atendidos no Rio de Janeiro resultou em custo médio anual igual a R\$3.211 (Araujo et al., 2005). Também realizamos atualização pelo IPCA, na qual o valor resultante foi de $\mathrm{R} \$ 6.225$.

Para AVC, não foi localizado artigo que tenha estimado diretamente os valores. No trabalho de Ribeiro et al., que avaliou o custo-efetividade de estatinas na prevenção de eventos vasculares, o valor anual de seguimento de AVC foi estimado em R\$ 1.033, por meio de painel de especialistas e alguns dados de seguimento de pacientes com AVC de Porto Alegre, RS. Atualizando esse valor pela inflação acumulada, o resultado atualizado é de R\$1.267 (Ribeiro et al., 2015).

Não localizamos artigos que tenham estimado o custo de doença renal sem necessidade de diálise. Porém, considerando a menor necessidade de tratamentos desses pacientes, e que os custos crônicos não dialíticos provavelmente possuem grande overlap com custos já contabilizados pelo seguimento crônico de insuficiência cardíaca e cardiopatia isquêmica, esses valores não foram computados no caso-base. Os custos crônicos utilizados no modelo são mostrados na Tabela 3.

O preço da empagliflozina considerou o valor da Câmara de Regulação do Mercado de Medicamentos (CMED) com preço fábrica e 18\% de ICMS, aplicando o coeficiente de adequação de preços, o que resultou em um valor mensal de

Tabela 3. Custos crônicos subsequentes a eventos agudos assumidos no modelo

\begin{tabular}{lcc}
\hline Condição clínica & $\begin{array}{c}\text { Custo crônico } \\
\text { anual }\end{array}$ & Referência \\
\hline Cardiopatia isquêmica & $\mathrm{R} \$ 5.424,00$ & $\begin{array}{c}\text { (Ribeiro et al., } \\
\text { 2005) }\end{array}$ \\
\hline Insuficiência cardíaca & $\mathrm{R} \$ 6.225,00$ & $\begin{array}{c}\text { (Araujo et al., } \\
\text { 2005) }\end{array}$ \\
\hline Insuficiência renal em diálise & $R \$ 37.184,00$ & $\begin{array}{c}\text { (Cherchiglia } \\
\text { et al., 2010) }\end{array}$ \\
\hline Acidente vascular cerebral & $\mathrm{R} \$ 1.267,00$ & $\begin{array}{c}\text { (Ribeiro et al., } \\
\text { 2015) }\end{array}$ \\
\hline
\end{tabular}

R\$ 134,75. O valor é idêntico tanto na apresentação de $10 \mathrm{mg}$ quanto de $25 \mathrm{mg}$.

Para infecção urinária, na ausência de estimativa mais acurada, utilizamos o valor recuperado no Datasus para tratamento de pielonefrite entre janeiro e dezembro de 2016, que foi de $R \$ 304,89$. Para infecção genital, que usualmente é fúngica, foi considerado o custo de um comprimido de fluconazol (preço médio no banco de preços em saúde de R\$ 0,20) e de uma consulta médica (R\$ 10, de acordo com o SIGTAP).

Finalmente, arbitramos um custo para a morte, conforme é feito comumente em modelos de doenças cardiovasculares, estimando que 50\% dos pacientes venham a óbito por eventos não súbitos, associados, portanto, a uma internação hospitalar. Foi realizada média aritmética dos custos de internação por ICC, AVC e IAM (considerando que essas eram as principais causas de morte cardiovascular no estudo EMPA-REG OUTCOME), e utilizado um valor igual a 50\% dessa média como custo para pacientes que vão a óbito ( $R \$ 1.268,66)$.

\section{Análises de sensibilidade}

Foram feitas as seguintes análises de sensibilidade determinísticas:

- Taxa de desconto, variada entre 0\% e 10\%;

- Efetividade: os hazard ratios de morte cardiovascular, internação por ICC e desfechos renais foram alterados dentro de seus IC 95\%, de forma simultânea;

- Valores de utilidade: variação simultânea de todos os valores em $\pm 20 \%$;

- Custos: variação simultânea dos custos de eventos agudos em $\pm 20 \%$; variação simultânea dos custos de crônicos de manutenção em $\pm 20 \%$; variação dos custos da empagliflozina em $\pm 20 \%$;

- Cenários alternativos de população-alvo, simulando coortes de apenas pacientes com: AVC prévio, IAM prévio e doença vascular periférica prévia. Além disso, também foi feita análise de cenário com população acima de 65 anos, uma vez que esse grupo apresentou, em análise de subgrupo do EMPA-REG OUTCOME, maior redução de eventos cardiovasculares que a população abaixo dessa faixa etária.

A análise de sensibilidade probabilística foi rodada com 1.000 simulações, cada uma com 1.000 pacientes.

\section{Resultados}

O modelo foi rodado na análise do caso-base com 15.000 pacientes. Os resultados encontrados são apresentados naTabela 4. O tratamento com empagliflozina resultou em 6,58 QALYs, 0,66 a mais do que o tratamento padrão, e 9,15 AVGs, equivalente a 0,95 além do gerado pelo tratamento padrão. Os custos totais do seguimento dos pacientes foram de 
Tabela 4. Resultados do caso-base

\begin{tabular}{lccccc}
\hline Intervenção & Custo total (R\$) & AVG & QALY & RCEI (R\$/AVG) & RCEI (R\$/QALY) \\
\hline Empagliflozina & $R \$ 65.289$ & 9,15 & 6,68 & - & - \\
\hline Tratamento padrão & $R \$ 46.031$ & 8,20 & 5,92 & - & - \\
\hline Incremental & $R \$ 19.176$ & 0,95 & 0,66 & $R \$ 20.185$ & $R \$ 28.960$ \\
\hline
\end{tabular}

AVGs: anos de vida ganhos; QALY: anos de vida ganhos ajustados pela qualidade; RCEl: razão de custo-efetividade incremental.

R\$ 65.289 na empagliflozina e de $\mathrm{R} \$ 46.113$ no tratamento padrão, gerando relações de custo-efetividade incremental de $R \$ 28.960$ por QALY e de R\$20.185 por AVG. Em termos de sobrevida global, sem aplicação da taxa de desconto, pacientes na empagliflozina viveram 13,64 anos, enquanto no tratamento padrão, 11,65 anos.

Os resultados da análise de sensibilidade determinística são apresentados na Tabela 5. Conforme pode ser visto, a RCEl se manteve robusta, atingindo o maior valor no cenário com decremento do valor de utilidade basal em 20\%. Em algumas outras situações, a RCEl também superou o valor de uma vez o Produto Interno Bruto (PIB) per capita (R\$30.407 em 2016). A análise de sensibilidade probabilística teve uma RCEl média de R\$29.842, com intervalo de credibilidade (percentis 2,5\% a 97,5\%) de $\mathrm{R} \$ 22.336$ a R\$39.571. O diagrama de dispersão das simulações é mostrado na Figura 2. O valor máximo das simulações foi de R\$ 54.328 por QALY.

\section{Discussão}

Neste estudo, realizamos uma análise de custo-efetividade de empagliflozina para o tratamento de pacientes com dia-

Tabela 5. Resultados da análise de sensibilidade determinística

\begin{tabular}{|c|c|c|}
\hline Parâmetros & RCEI mínima & RCEI máxima \\
\hline Taxa de desconto & $\mathrm{R} \$ 23.982$ & $R \$ 35.525$ \\
\hline Hazard ratios de efetividade & $R \$ 24.589$ & $R \$ 33.954$ \\
\hline Valor de empagliflozina & $\mathrm{R} \$ 24.491$ & $\mathrm{R} \$ 33.405$ \\
\hline Custos dos eventos agudos & $R \$ 28.903$ & $R \$ 29.010$ \\
\hline Custos dos eventos crônicos & $R \$ 27.067$ & $R \$ 29.718$ \\
\hline Valor de utilidade basal & $R \$ 23.715$ & $R \$ 37.186$ \\
\hline $\begin{array}{l}\text { Decrementos de utilidade } \\
\text { associados a eventos }\end{array}$ & $\mathrm{R} \$ 28.6211$ & $\mathrm{R} \$ 28.967$ \\
\hline $\begin{array}{l}\text { Cenário: apenas pacientes } \\
\text { com AVC prévio }\end{array}$ & \multicolumn{2}{|c|}{$R \$ 39.608$} \\
\hline $\begin{array}{l}\text { Cenário: apenas pacientes } \\
\text { com IAM prévio }\end{array}$ & \multicolumn{2}{|c|}{$R \$ 39.986$} \\
\hline $\begin{array}{l}\text { Cenário: apenas pacientes } \\
\text { com DVP prévia }\end{array}$ & \multicolumn{2}{|c|}{$R \$ 19.240$} \\
\hline $\begin{array}{l}\text { Cenário: população com } \\
\text { mais de } 65 \text { anos }\end{array}$ & \multicolumn{2}{|c|}{$R \$ 30.505$} \\
\hline
\end{tabular}

AVC: acidente vascular cerebral; DVP: doença vascular periférica; IAM: infarto agudo do miocárdio; RCEl: razão de custo-efetividade incremental. betes e alto risco cardiovascular, definido pela ocorrência de eventos vasculares prévios (IAM, AVC ou doença vascular periférica). A relação de custo-efetividade incremental no caso-base foi de R\$29.190 por QALY, o que fica abaixo de uma vez o PIB per capita brasileiro. Ainda que não haja aceitação oficial do limiar sugerido pela comissão de macroeconomia da Organização Mundial da Saúde, no qual valores abaixo de uma vez o PIB per capita de um país eram vistos como bastante custo-efetivos e abaixo de três vezes o PIB per capita, como custo-efetivos (WHO, 2001), esses valores são frequentemente empregados em estudos de custo-efetividade conduzidos no Brasil (Schwarzer et al., 2015). Nas análises de sensibilidade, a incerteza acerca da estimativa de alguns parâmetros resultou em uma RCEI acima desse limiar de uma vez o PIB per capita; porém, tanto nas análises determinísticas como no percentil 97,5\% da análise probabilística, a extrapolação de tal valor não foi muito grande, com as estimativas se situando abaixo de 1,3 vez o PIB per capita. Desse modo, e especialmente comparando com outras tecnologias recentemente incorporadas no SUS, as quais tiveram valores de custo-efetividade acima dessa cifra - fingolimode para esclerose múltipla, com RCEl de R\$77.095,00 (Conitec, 2017), e levetiracetam para epilepsia, com RCEl de R\$58.294,00 (Conitec, 2017), entendemos que os resultados são robustos, uma vez que 100\% dos valores de RCEI na análise probabilística (que espe-

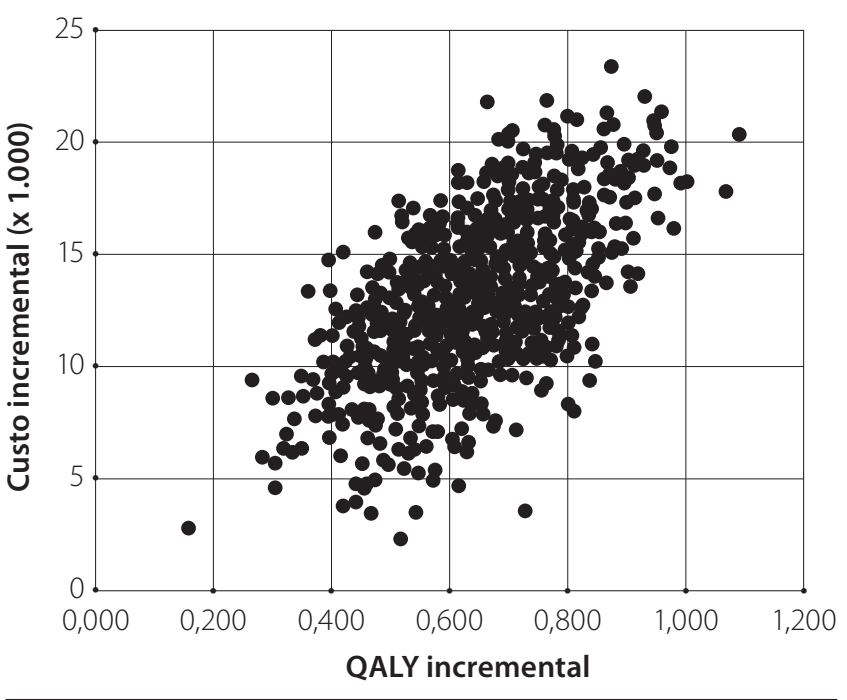

Figura 2. Diagrama de dispersão da análise de sensibilidade probabilística. 
Iham, portanto, toda a incerteza nos parâmetros do modelo) se situam abaixo desses valores de outras incorporações.

Na literatura internacional, ainda existem poucos estudos de custo-efetividade da empagliflozina publicados na forma de manuscritos completos. Em trabalho realizado por pesquisadores vinculados a universidades americanas, a RCEI da empagliflozina versus tratamento padrão em diabéticos com alto risco cardiovascular foi de US\$ 76.167 por QALY, se situando, em 96\% das simulações na análise probabilística, abaixo do limiar sugerido pelos pesquisadores de US\$100.000 por QALY. Já em estudo grego, com população e comparação semelhante, a RCEI foi muito mais baixa, de apenas $€ 4.633$ por QALY. Realizar um adequado contraponto dos resultados econômicos do nosso estudo com dados internacionais é uma tarefa difícil, uma vez que a variação de custos entre diferentes países é muito grande. Porém, os dados de efetividade são mais comparáveis, e a estimativa feita em nosso estudo é conservadora, em relação à literatura internacional: em nosso modelo, o ganho em QALYs foi de 0,65; no estudo americano, foi de 1,30; e no grego, de 0,92.

Algumas limitações do presente estudo devem ser mencionadas. O seguimento do ensaio clínico que norteia o modelo é de 3,1 anos, portanto menor que o horizonte temporal do modelo, que foi de tempo de vida, equivalente a 13,6 e 11,6 anos para a empagliflozina e para o tratamento padrão, respectivamente. Apesar de ser uma limitação, é bastante comum em estudos de custo-efetividade de medicamentos que a efetividade seja mantida constante ao longo do tempo. Não é possível prever exatamente qual o impacto desse pressuposto nos resultados; caso o benefício da empagliflozina na prática se dilua ao longo do tempo, o modelo atual estaria favorecendo a empagliflozina. Outra limitação do estudo é a utilização de dados de utilidade internacionais para três das condições de saúde modeladas. Porém, para os quatro desfechos que possuíamos dados nacionais, os valores foram muito próximos ao estudo internacional que balizou os demais dados, sugerindo que essa limitação teve impacto mínimo nos resultados. Ainda sobre os dados de utilidade, foi usado valor de decremento igual nas condições renais (isto é, macroalbuminúria, insuficiência renal não dialítica e dialítica), pois o estudo que provê os dados não realizou distinção entre os diferentes estágios de nefropatia. Provavelmente, a perda de utilidade da diálise seria mais expressiva do que o resultado utilizado no modelo. Como a empagliflozina reduz significativamente o número de pacientes que evoluem para diálise, essa limitação acaba sendo conservadora, provavelmente reduzindo a custo-efetividade da empagliflozina. Outro ponto a salientar como limitação foi o não cômputo de custos agudos e crônicos de doença renal não dialítica e de macroalbuminúria, tendo em vista a ausência de estimativas consistentes. Porém, tais custos provavelmente são diferentes de zero, pois alguns exames de seguimento adicionais são feitos nesses pacientes. Como a incidência de tais eventos é maior no grupo do tratamento padrão, essa limitação também é conservadora.

Por outro lado, também é importante salientar as três principais virtudes do presente estudo. Primeiramente, a fonte de dados de efetividade é bastante robusta, oriunda de ensaio clínico randomizado com mais de 7.000 pacientes e que aferiu diretamente desfechos clinicamente relevantes. Em segundo lugar, o modelo empregado foi robusto, e utilizou diretamente os dados individuais dos pacientes do ensaio clínico referido. Por último, para a maioria dos parâmetros, foram obtidas estimativas de valores de utilidade de estudos nacionais, o que é pouco frequente em estudos de custo-efetividade no Brasil.

Em conclusão, o presente trabalho mostrou que a empagliflozina é um tratamento potencialmente custo-efetivo para pacientes com DM2 e alto risco cardiovascular, com resultados robustos em relação às incertezas dos parâmetros. Ainda que estudos de custo-efetividade não devam ser os únicos norteadores de tomada de decisão, a qual deve levar em consideração também outros aspectos como impacto orçamentário e equidade, entre outros, o presente trabalho fornece evidência de qualidade para o processo decisório.

\section{Referências bibliográficas}

Araujo DV, Tavares LR, Veríssimo R, Ferraz MB, Mesquita ET. [Cost of heart failure in the Unified Health System]. Arq Bras Cardiol. 2005;84(5):422-7.

Bahia LR, Araujo DV, Schaan BD, Dib SA, Negrato CA, Leão MP, et al. The costs of type 2 diabetes mellitus outpatient care in the Brazilian public health system. Value Health. 2011;14(5 Suppl 1):S137-40.

Braga SFM, Peixoto SV, Gomes IC, Acúrcio FA, Andrade EIG, Cherchiglia ML. Factors associated with health-related quality of life in elderly patients on hemodialysis. Rev Saúde Pública. 2011;45(6):1127-36.

Cherchiglia ML, Gomes IC, Alvares J, Guerra Júnior A, Acúrcio FA, Andrade EIG, et al. [Determinants of expenditures on dialysis in the Unified National Health System, Brazil, 2000 to 2004]. Cad Saúde Pública. 2010;26(8):1627-41.

Conitec. Fingolimode no tratamento da esclerose múltipla remitente recorrente após falha terapêutica com betainterferona ou glatirâmer. Brasília: Ministério da Saúde; 2017.

Cruz LN, Camey SA, Fleck MP, Polanczyk CA. World Health Organization quality of life instrument-brief and Short Form-36 in patients with coronary artery disease: do they measure similar quality of life concepts? Psychol Health Med. 2009;14(5):619-28.

Cruz LN, Camey SA, Hoffmann JF, Rowen D, Brazier JE, Fleck MP, et al. Estimating the SF-6D value set for a population-based sample of Brazilians. Value Health. 2011;14(5 Suppl 1):S108-14.

de Abreu MM, Walker DR, Sesso RC, Ferraz MB. Health-related quality of life of patients recieving hemodialysis and peritoneal dialysis in São Paulo, Brazil: a longitudinal study. Value Health. 2011;14(5 Suppl 1):S119-21. 
Favarato ME, Hueb W, Boden WE, Lopes N, Nogueira CR, Takiuti M, et al. Quality of life in patients with symptomatic multivessel coronary artery disease: a comparative post hoc analyses of medical, angioplasty or surgical strategies-MASS II trial. Int J Cardiol. 2007;116(3):364-70.

Ferreira KA. Análise de custo-efetividade de procedimentos para o adiamento da fase terminal da doença renal crônica associada ao diabetes mellitus e à hipertensão arterial sob a perspectiva do Sistema Único de Saúde [dissertação]. Rio de Janeiro: Escola Nacional de Saúde Pública Sergio Arouca; 2009.

Iser BPM, Stopa SR, Chueiri PS, Szwarcwald CL, Malta DC, Monteiro HOC, et al. Prevalência de diabetes autorreferido no Brasil: resultados da Pesquisa Nacional de Saúde 2013. Epidemiol Serv Saúde. 2015;24(2):305-14.

Lima MG, Barros MBA, Alves MCGP. Sleep duration and health status selfassessment (SF-36) in the elderly: a population-based study (ISA-Camp 2008). Cad Saúde Pública. 2012;28(9):1674-84.

Moreira DM, Vieira JL, Gottschall CA. The effects of METhotrexate therapy on the physical capacity of patients with ISchemic heart failure: a randomized double-blind, placebo-controlled trial (METIS trial). J Card Fail. 2009;15(10):828-34.

Ribeiro RA, Duncan BB, Ziegelmann PK, Stella SF, Vieira JLC, Restelatto LMF, et al. Cost-Effectiveness of High, Moderate and Low-Dose Statins in the Prevention of Vascular Events in the Brazilian Public Health System. Arq Bras Cardiol. 2015;104(1):32-44.

Ribeiro RA, Mello RGB, Melchior R, Dill JC, Hohmann CB, Lucchese AM, et al. Custo anual do manejo da cardiopatia isquêmica crônica no Brasil. Perspectiva pública e privada. Arq Bras Cardiol. 2005;85(1):3-8.
Ribeiro RA, Neyeloff JL, Itria A, Santos VCC, Vianna CMM, Silva EN, et al. Diretriz metodológica para estudos de avaliação econômica de tecnologias em saúde no Brasil. J Bras Econ Saúde 2016;8(3):174-84

Rosa R, Nita ME, Rached R, Donato B, Rahal E. Estimated hospitalizations attributable to Diabetes Mellitus within the public healthcare system in Brazil from 2008 to 2010: study DIAPS 79. Rev Assoc Med Bras. 2014;60(3):222-30.

Saccomann ICRS, Cintra FA, Gallani MCBJ. Health-related quality of life among the elderly with heart failure: a generic measurement. Sao Paulo Med J. 2010;128(4):192-6.

Santos M, Cintra MA, Monteiro AL, Santos B, Gusmão-Filho F, Andrade MV, et al. Brazilian Valuation of EQ-5D-3L Health States: Results from a Saturation Study. Med Decis Making. 2016;36(2):253-63.

Schwarzer R, Rochau U, Saverno K, Jahn B, Bornschein B, Muehlberger N, et al. Systematic overview of cost-effectiveness thresholds in ten countries across four continents. J Comp Eff Res. 2015;4(5):485-504.

Sesso R, Rodrigues-Neto JF, Ferraz MB. Impact of socioeconomic status on the quality of life of ESRD patients. Am J Kidney Dis. 2003;41(1):186-95.

Sullivan PW, Ghushchyan VH. EQ-5D Scores for Diabetes-Related Comorbidities. Value Health. 2016;19(8):1002-8.

WHO. Macroeconomics and health: investing in health for economic development: report of the commission on macroeconomics and health. Geneva: World Health Organization; 2001.

Zinman B, Wanner C, Lachin JM, Fitchett D, Bluhmki E, Hantel S, et al. Empagliflozin, cardiovascular outcomes, and mortality in type 2 diabetes. N Engl J Med. 2015;373(22):2117-28. 